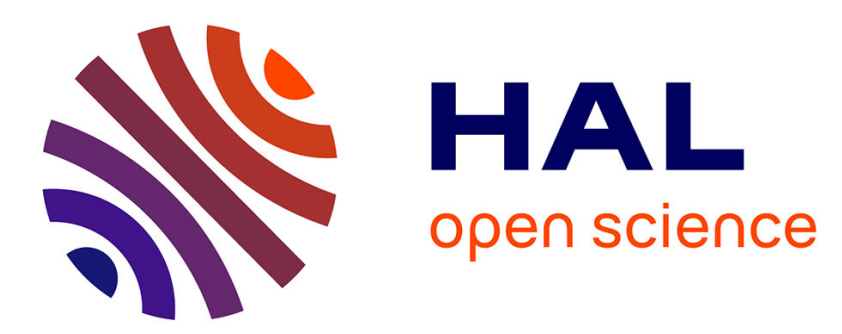

\title{
Maternal condition determines offspring behavior toward family members in the European earwig
}

\author{
Jos Kramer, Joël Meunier
}

\section{To cite this version:}

Jos Kramer, Joël Meunier. Maternal condition determines offspring behavior toward family members in the European earwig. Behavioral Ecology, 2016, 27 (2), pp.494-500. 10.1093/beheco/arv181 . hal-02117966

\section{HAL Id: hal-02117966 \\ https://hal-univ-tours.archives-ouvertes.fr/hal-02117966}

Submitted on 11 Jun 2020

HAL is a multi-disciplinary open access archive for the deposit and dissemination of scientific research documents, whether they are published or not. The documents may come from teaching and research institutions in France or abroad, or from public or private research centers.
L'archive ouverte pluridisciplinaire HAL, est destinée au dépôt et à la diffusion de documents scientifiques de niveau recherche, publiés ou non, émanant des établissements d'enseignement et de recherche français ou étrangers, des laboratoires publics ou privés. 
Maternal condition determines offspring behavior towards family members in the European earwig

Running title: Maternal condition and family interactions in earwigs

Jos Kramer ${ }^{1 *}$ and Joël Meunier ${ }^{1}$

${ }^{1}$ Department of Evolutionary Biology, Institute of Zoology, Johannes Gutenberg University of Mainz, Mainz, Germany.

*Corresponding author: J. Kramer, JosKramer@gmx.de

Email addresses and phone numbers: J. Kramer (JosKramer@gmx.de; phone: +49 6131 - 39 27853; fax: +49 6131 - 39 27850); J. Meunier (meunier.joel@gmail.com; phone: +49 6131 39 27852)

\section{LAY SUMMARY}

The condition of parents does not only influence parental behavior, but also determines how their offspring interact with each other. By manipulating the nutritional state of tending females, we showed in the European earwig that maternal condition affected the food transfer among their offspring and the begging behavior these juveniles direct towards their mother. Importantly, the body condition of mothers also influences their own maternal behavior. 


\section{ABSTRACT}

Parental care confers benefits to juveniles, but is usually associated with substantial costs for parents. These costs often depend on parental condition, which is thus considered as a key determinant of the level of parental care expressed during family life. However, how parental condition affects the behaviors that juveniles express towards their siblings and parents remains poorly explored. Here, we investigated these questions in the European earwig Forficula auricularia, an insect in which mothers provide extensive forms of care to their juveniles. We measured maternal body condition at egg hatching, subsequently manipulated maternal nutritional state and finally assessed both food transfer among siblings and the nature of mother-offspring interactions. We also considered variation in brood size, an important parameter in family interactions. We found that food transfer among siblings increased with brood size when the tending mothers were in a deteriorated nutritional state. This effect was masked when the nutritional state of mothers was enhanced. The frequency of care-related behaviors that juveniles expressed towards their mother was higher when she was in a deteriorated rather than an enhanced nutritional state, while it overall increased with brood size. Finally, increasing values of maternal body condition entailed a shift from a positive to a negative association between maternal care behaviors and brood size, but only when the mothers' nutritional state was deteriorated. Overall, our results demonstrate that parental condition and brood size do not only affect parental behaviors, but can also be important and entangled drivers of offspring behaviors during family life. 
Keywords: social evolution; precocial species; parental care; sibling rivalry; Forficula auricularia 


\section{INTRODUCTION}

Parental care is a common and taxonomically widespread phenomenon in nature and usually confers substantial benefits to the tended juveniles (Royle et al., 2012). However, providing care often entails high costs for parents, such as an increased loss of energy or an elevated risks of infection and predation (Alonso-Alvarez and Velando 2012), which can reduce their ability to invest in future reproduction (Trivers 1972). Since parents in a bad condition are expected to incur higher costs of care than parents in a good condition (Hinde et al. 2010), they are generally predicted to adjust the expression of parental care to their body condition as well as to short-term changes in their nutritional state in order to maximize their reproductive success (Bateson 1994). Several studies provided support for this prediction (e.g. Markman et al. 2002; Gorman and Nager 2003; Laurien-Kehnen and Trillmich 2004; Bleeker et al. 2005; Segers et al. 2011; Wong and Kölliker 2012), thus revealing the central importance of parental body condition and nutritional state in the expression of parental care. For instance, mothers in a poor nutritional state were shown to reduce parental care towards their current offspring in the mouthbrooding cichlid Simochromis pleurospilus (Segers et al. 2011), whereas food-deprived guinea pig (Cavia porcellus) mothers prolonged the expression of nursing behavior while maintaining a constant body condition, thus ultimately reducing the growth rate of their pups (LaurienKehnen and Trillmich 2004). Conversely, artificial food supplementation with a sucrose solution allowed parent palestine sunbirds (Nectarinia osea) to increase the rate at which they provisioned their nestlings with arthropods (Markman et al. 2002).

The optimal level of care from the point of view of the parents, however, does not necessarily coincide with the optimal level of care from the offspring's point of view. 
Because of relatedness asymmetries among family members, offspring often behave more selfishly than their parents desire (Trivers 1974; see also Mock and Forbes 1992), both by trying to monopolize resources at the expense of their siblings (sibling rivalry; Mock and Parker 1997; Roulin and Dreiss 2012), as well as by manipulating their parents into increasing their parental investment (Kilner \& Hinde, 2012). The resulting parent-offspring conflict over the amount of parental investment into care compels parents and offspring to monitor each other's state (Royle et al. 2002; Morales and Velando 2013) and to adjust their strategy of providing and demanding care accordingly (e.g. Godfray and Johnstone 2000; Parker et al. 2002; Smiseth et al. 2003). By analogy, parental body condition and nutritional state should not only influence the behavior of tending parents, but might also affect how offspring interact with each other, as well as how they act towards their parents (Bateson 1994).

While a number of studies have tested the influence of parental body condition on the expression of parental care and the (begging) behavior of offspring towards their parents (see above), it remains poorly explored how parental condition and its changes during family life affect the behavior of juveniles towards their own siblings (but see White et al. 2010; Wong, Lucas, et al. 2014 for effects on aggressive behavior). Here, we investigated this effect in the European earwig F. auricularia. In this insect species, mothers provide non-obligatory forms of care to their mobile offspring (called nymphs) for several weeks after hatching (Lamb 1976 a). Maternal care comprises multiple behaviors including the provisioning of nymphs (Lamb 1976 b; Staerkle and Kölliker 2008), the amount of which is reduced when mothers had limited access to food resources during family life (Wong and Kölliker 2012). While such condition-dependent behavioral changes in maternal care might 
enable nymphs to indirectly monitor short-term changes in their mother's condition, nymphs can also assess maternal condition based on cues/signals that are encoded in the profile of the mother's cuticular hydrocarbons (Wong, Lucas, et al. 2014). Interestingly, maternal presence and post-hatching care are not obligatory for offspring survival (Lamb 1976 b; Kölliker 2007; Kölliker and Vancassel 2007). Earwig nymphs do not exclusively rely on maternal provisioning, but may also forage independently soon after hatching (Lamb 1976 b; Wong and Kölliker 2012) and obtain food from their siblings (Falk et al. 2014; Kramer et al. 2015). This food transfer among juveniles is predominantly mediated by active allo-coprophagy, a process defined as a socially-induced increase in feces production by donor nymphs and the subsequent consumption of these feces by recipient siblings (Falk et al. 2014).

To unravel whether offspring behaviors towards family members were associated with maternal condition at egg hatching (initial body condition; a proxy for the mother's long-term energetic state) and/or the post-hatching access of mothers to food resources (nutritional state; a proxy for short-term changes in the satiety level), we first determined the body condition of mothers at egg hatching, subsequently manipulated their nutritional state during four days and then assessed sibling food transfer and mother-offspring interactions. If the mother's nutritional state influenced the behavior of offspring towards their siblings and towards their mother, we would expect (1) a decrease in food transfer among the nymphs, as well as (2) a lower frequency of care-related offspring behaviors (such as begging) in families tended by mothers in an enhanced as compared to a deteriorated nutritional state. This is because mothers in an enhanced nutritional state typically show higher levels of maternal care (Wong and Kölliker 2012). We also expected 
that mothers with a high body condition at egg hatching could generally afford higher levels of care than mothers with a low initial body condition. Accordingly, we predicted that (3) a high maternal body condition at egg hatching would reinforce the positive effects of an enhanced nutritional state on the level of parental care and (4) allow, in comparison to a low initial body condition, higher levels of care when a mother's current state is deteriorated. Note that we also considered (differences in) family size in our analyses of the effects of maternal condition and nutritional state on family interactions, because group size is generally assumed to affect the competition among group members (Alexander 1974; Shen et al. 2014) and could thus modify reactions of family members to changes in maternal condition or state. Moreover, family size has been suggested to influence mortality and developmental rates of nymphs in European earwigs (Kölliker 2007; Meunier et al. 2012; Meunier and Kölliker 2012) and is linked to the level of food transfer among their offspring (Kramer et al. 2015).

\section{MATERIALS AND METHODS}

\section{(a) Study animals and laboratory rearing}

The adult $F$. auricularia females used in the experiment descended from 80 female and 73 male earwigs collected in September 2012 in a natural population in Dolcedo, Italy. These individuals were maintained in the laboratory under standard rearing conditions (detailed in Koch and Meunier 2014) for two generations. The experiment comprised of two randomly chosen, independent subsets of adult females and their respective first brood of offspring derived from the first ( $n=19$ families used in 2013) and the second $(n=29$ families used in 2014) filial generation, respectively. These subsets neither differed with respect to maternal 
body size, weight or body condition at egg hatching (details on these measurements below) nor with regards to brood size (see Manova results below).

\section{(b) Experimental setup}

A total of 48 families were used to disentangle the effects of maternal body condition at egg hatching and subsequent changes in maternal nutritional state on the level of sibling food transfer and the frequency of mother-offspring interactions. The corresponding experimental design is detailed in Figure 1. Females had no access to food between egg laying and hatching (mean duration \pm se: $33 \pm 2$ days), as $F$. auricularia females generally do not consume food during that period of time (Kölliker 2007). One day after egg hatching, families were randomly attributed to one of two treatments. In the first treatment $(n=24$ families; 9 in 2013 and 15 in 2014), mothers did not receive any food before the behavioral observations (i.e. until day four, deteriorated state). In the second treatment, mothers had access to uncolored food (naturally yellow colored flower pollen formed into pellets; Hochland Bio-Blütenpollen, Hoyer $\mathrm{GmbH}$, Polling, Germany) for 20 minutes on the first and the fourth day after hatching (enhanced state; $n=23$ families; 9 in 2013 and 14 in 2014). As intended, the nutritional state of females in the first treatment deteriorated, whereas the state of females in second treatment improved (see details in the supplementary material). In both treatments, nymphs were provided with uncolored food for 1.5 hours on the first and second day after hatching. No food was provided on the third day after hatching to increase their foraging and solicitation behavior towards mothers and siblings on the following day (Staerkle and Kölliker 2008; Falk et al. 2014). On day four after hatching, the 
nature and frequency of mother-offspring interactions as well as the level of sibling food transfer were investigated in each family (see details below).

Throughout the experiment, families were kept in their original, medium-sized Petridish $(9 \times 2 \mathrm{~cm})$ with humid sand as ground material and a plastic tube as shelter. During offspring feeding and (where applicable) to provide them with food, mothers were isolated in a small Petri dish $(5.5 \times 1.2 \mathrm{~cm})$ that only contained humid sand as ground material. Brood size was established by counting the number of nymphs one day after the first egg hatching had been observed. To control that our treatments induced changes in maternal nutritional state, females were weighed on the first and fourth day after hatching (before the onset of behavioral observations) to the nearest $0.01 \mathrm{mg}$ using a micro-scale (model MYA5; PESCALE, Bisingen, Germany). After the behavioral observations, females were sedated with $\mathrm{CO}_{2}$ and their eye distance was measured to the nearest $0.001 \mathrm{~cm}$ using a camera coupled to a binocular (Leica DFC425, Leica Microsystems Ltd., Heerbrugg, Switzerland) and the software Leica Application Suite 4.5.0. Using eye distance as a measure of body length together with female weight at egg hatching, we calculated the initial body condition for each female based on the 'scaled mass index' (Peig and Green 2009; Peig and Green 2010). In brief, this index standardizes body mass at a fixed value of a linear body measurement based on the scaling relationship between these measures (Peig and Green 2009). Accordingly, this index indicates which mass a particular female would have at the average eye distance. Note that initial body condition, brood size, as well as maternal weight and size at egg hatching did not differ between the 'enhanced state' and 'deteriorated state' treatments in both experimental seasons (Manova; interaction treatment: season: $\Lambda_{\text {Pillai, } 1}=0.052, P=0.700$; treatment: $\Lambda_{\text {Pillai, } 1}=0.032, \mathrm{P}=0.848$; season: $\left.\Lambda_{\text {Pillai, } 1}=0.106, \mathrm{P}=0.308\right)$. 


\section{(c) Assessments of sibling food transfer and mother-offspring interactions}

The measurement of sibling food transfer relied on the fact that ingested colored food remains visible through the partially transparent cuticle of young $F$. auricularia nymphs (Staerkle and Kölliker 2008; Falk et al. 2014). Sibling food transfer was measured in three successive steps according to a previously established, standard protocol (Kramer et al. 2015). First, we provided one half of the nymphs (called donor nymphs) with colored food (naturally yellow colored pollen mixed with a blue food dye; Dekoback, Online Ideen GmbH, Germany) for $1 \mathrm{~h}$ in a small Petri dish, while the remaining nymphs were starved separately. Simultaneously, mothers were either starved or had access to uncolored pollen for 20 min (see treatments above). Note that temporary separations of nymphs from their mother commonly occur in nature during independent foraging trips (Lamb $1976 \mathrm{~b}$ ). In the second step, we reassembled the newly colored donor nymphs with their remaining siblings (called recipient nymphs) and their mother to allow family interactions. Fifteen hours later, we finally counted the number of recipient nymphs that ingested the colored food provided by the donor nymphs using a stereo-microscope. To be able to discriminate between donors and recipients nymphs, either all donor ( $n=27$ trials) or all recipient nymphs ( $n=20$ trials) of a given family were chosen at random and marked by clipping off the distal third of their right cercus (Wong and Kölliker 2013) prior to the experiment. Nymphal coloration was scored by one single observer and blindly with regard to the treatment of the mother throughout the experiment. The level of sibling food transfer (measured as the proportion of recipient nymphs that received colored food from siblings) was independent of marking (Wilcoxon rank sum test; $\mathrm{W}=263, \mathrm{P}=0.889$ ). 
The behavioral interactions between mothers and their offspring were assessed in the course of the measurement of sibling food transfer and categorized into care-related behaviors expressed by offspring, but also care and aggressive behaviors expressed by mothers. The behaviors were recorded using a scan sampling approach (one observation every 5 min for $45 \mathrm{~min}$, i.e. 10 scans in total per replicate) starting $15 \mathrm{~min}$ after the family members had been re-assembled in their original Petri dish to allow food transfer (see above). Care-related offspring behaviors comprised (1) 'licking' behaviors, during which nymphs manipulate the inter-segmental skin between abdominal segments and/or the legjoints of the mother with their mouthparts, (2) begging behaviors, during which nymphs try to establish mouth-to-mouth-contact with the female and (3) mouth-to-mouth contacts. The number of occurrences of each of these behaviors (i.e. the number of times a given behavior was performed by at least one nymph) was then summed up across all ten scans obtain the overall frequency of care-related offspring behaviors. Maternal care behavior was defined following Mas \& Kölliker (2011) and was recorded as the sum of (1) antennal contacts with the nymphs, (2) allo-grooming during which the mother manipulated nymphs with her mouth parts and (3) mouth-to-mouth contacts. Mouth-to-mouth contact was considered as both female and nymph behavior because it required coaction of the interacting individuals. Finally, aggressive behaviors expressed by mothers were the sum of threat displays, during which the female raised her forceps in the direction of a nymph, and abdomen shaking, a behavior allowing females to cast off riding nymphs. As this study only focuses on mother-offspring interactions, we did not analyze self-directed female behaviors such as resting, self-grooming and exploring. All behavioral observations were conducted blindly with respect to the nutritional state of the female. Note that all of the above detailed measurements were based on half of the nymphs per family (half of the nymphs were 
haphazardly chosen and removed from their families before the onset of the observations reported in this study. These nymphs were used in a different experiment; data not shown).

\section{(d) Statistical analyses}

The effects of initial body condition, offspring number and nutritional state on sibling food transfer and mother-offspring interactions were tested in three Generalized Linear Models (GLMs). In these models, initial body condition (continuous), brood size (continuous), nutritional state (enhanced or deteriorated; bimodal) and all their interactions were entered as explanatory variables. As the experiment was conducted in two consecutive seasons, we additionally entered 'season' as a bimodal (2013 and 2014) explanatory variable in all models to account for potential differences caused by this confounding factor. We initially also included the interaction between 'season' and 'nutritional state' into our models, but subsequently removed it because it was never significant (all $P<0.128$ ). The frequency of maternal care behaviors and the relative frequency of care-related nymphal behaviors were analyzed in two separate GLMs with a poisson error distribution corrected for overdispersion. We used the relative instead of the absolute frequency of care-related nymphal behaviors (i.e. the absolute frequency divided by the brood size) to avoid a potentially confounding effect of differences in nymph number on this measure of offspring behavior. The proportion of recipient nymphs colored after the sibling food transfer test (entered as odds ratio using the 'cbind'-function in R) was analyzed in a GLM with binomial error distribution corrected for overdispersion. Note that we did not statistically analyze the frequency of female aggressions against her offspring, as such aggressions occurred 
infrequently, both within broods (none of the 47 families featured more than 1 aggression) and across broods (aggressive behavior was only observed in 5 of 47 families).

All statistical analyses were performed using the statistics software $\mathrm{R}$ 3.0.3 (http://www.r-project.org/). Mixed model analyses were implemented using the package Ime4. Significance levels of effects in these models were assessed using the packages car (Anova-function) and ImerTest (summary-function). Note that we centered 'brood size' and 'initial body condition' on their mean to avoid any model bias due to collinearity between these explanatory variables (variance inflation factor (VIF) $<5.5$ after centering in all models). All statistical models were simplified stepwise by removing non-significant interaction terms (all $\mathrm{P}>0.187$ ), as retaining these terms can bias estimates of other effects in the model (Engqvist 2005). A log-Likelihood Ratio test was used to test the explanatory power of each model after the removal of a variable. Where applicable, models were checked for normality of residuals and homogeneity of variance before and after the model selection procedure. Finally, interactions between continuous variables were plotted using the package effects to display the predicted relationship between the response variable and one explanatory variable for different, fixed values of the interacting variable(s) (details in Fox 2003).

\section{RESULTS}

Maternal nutritional state influenced the exchange of food among her offspring, but only through an interaction with brood size (Figure 2; Table 1A). Specifically, sibling food transfer increased with brood size when the mother's nutritional state was deteriorated (model estimate \pm se: $\left.0.048 \pm 0.016, t_{46}=3.073, P=0.004\right)$, whereas this association disappeared 
when maternal nutritional state was enhanced (estimate \pm se: $-0.0003 \pm 0.0163, t_{46}=-0.021$, $P=0.983)$. Overall, the level of food transfer did not differ among clutches that had been tended by females in a deteriorated or enhanced state, respectively (table $1 \mathrm{~A}$ ). Notably, sibling food transfer was independent of the initial body condition of the mother (table $1 \mathrm{~A}$ ).

The relative frequency of care-related behaviors expressed by nymphs was overall higher when mothers had a deteriorated compared to an enhanced nutritional state (Figure $3 A$; Table 1B), but was independent of brood size, the initial body condition of the mother and any interactions between the three tested variables (Table 1B). In contrast, a triple interaction between the initial body condition of the mother, brood size and her nutritional state influenced the frequency of care-related behaviors expressed by mothers (Table 1C). When the nutritional state of mothers was deteriorated, the frequency of care behaviors increased with brood size if they initially had been in bad condition, but surprisingly decreased with brood size if they had been in good condition (Figure 4; interaction between initial body condition and brood size: Likelihood ratio (LR) $\chi_{1}{ }^{2}=5.35, P=0.021$; initial body condition: $L R \chi_{1}{ }^{2}=0.97, P=0.325$; brood size: $\left.L R \chi_{1}^{2}=0.30, P=0.583\right)$. By contrast, when the nutritional state of mothers was enhanced, their initial body condition did not influence the frequency of maternal care behaviors, irrespective of brood size (interaction between initial body condition and brood size: $L R \chi_{1}^{2}=1.74, P=0.187$; initial body condition: $L R \chi_{1}{ }^{2}=$ 1.08, $P=0.298 ;$ brood size: $L R \chi_{1}{ }^{2}=3.26, P=0.071$ ). Finally, aggressive behavior of mothers against their offspring overall occurred infrequently and were distributed across seasons and experimental treatments without any obvious pattern (occurrences per family; 'enhanced state': $2014=0,2013=2$; 'deteriorated state': $2014=2,2013=1$ ). Note that season (2014 or 2013) never had a significant effect in any of our models (Table 1). 


\section{DISCUSSION}

In this study, we showed that behavioral interactions among family members in the European earwig F. auricularia reflect an interplay of the mother's current nutritional state, her condition at offspring emergence and the number of her offspring. Our data revealed that sibling food transfer increased with brood size when the female was in a bad nutritional state, but was independent of brood size when she was in an enhanced nutritional state. The level of sibling food transfer was, however, not linked to the female's condition at egg hatching. We also found that the expression of care-related behaviors by offspring was overall higher if the mother was in a deteriorated rather than an enhanced nutritional state. Finally, regarding maternal care, our results demonstrated that the frequency of caring behaviors - but likely not the rare occurrence of aggressions - was shaped by an interaction between the current state of the mother, her body condition at egg hatching and brood size. If the mother was in a deteriorated state at the time of measurement, the frequency of maternal care behaviors increased with brood size if she had also been in a bad condition at egg hatching, but surprisingly decreased with brood size when she had been in a good initial condition. Notably, this interactive effect was masked when the female was in an enhanced nutritional state.

Our results are in line with the prediction that a mother's nutritional state shapes the behavioral interactions among her offspring. When mothers were in a deteriorated state (and thus unable to provision their offspring), the increase of sibling food transfer with brood size could reflect a density-dependent increase of competition among recipients for publicly available feces and/or brood-size dependent changes in the propensity of donors to 
produce these feces (Kramer et al. 2015). Conversely, the absence of such an association when mothers were in an enhanced state and thus able to provision their offspring might reflect that females provided sufficient levels of care to limit selfishness among offspring that react to cues of female condition (Wong, Lucas, et al. 2014; Wong, Meunier, et al. 2014) and/or to mask the above described density-dependent effects. Interestingly, such a masking effect has recently been described in the burying beetle Nicrophorus vespilloides, where a density-dependent shift from cooperation to competition was only evident in the absence of parental care (Schrader et al. 2015). Further experiments are needed to differentiate among the above possibilities in earwigs. Nevertheless, the deteriorated state of mothers is unlikely to have specifically triggered a density-dependent increase of feces production by donor nymphs, as the overall level of sibling food transfer was found to be independent of female state. Instead, a deteriorated maternal state is likely to increase the competition among recipient nymphs, which might in turn reduce (rather than increase) the feces production of donor nymphs. The fact that the differences in the level of food transfer between treatments were most pronounced in small broods suggests that the reduction in feces production might be larger in these broods. Hence, while sibling food transfer can possibly mitigate detrimental effects of low maternal care by providing access to additional resources in the form of feces (Kramer et al. 2015), its extent might ultimately be limited because donor nymphs neither seem to adjust the production of feces directly to their siblings' nutritional need (Falk et al., 2014), nor indirectly by monitoring and reacting to their mother's condition (this study).

The frequency of care-related behaviors that offspring directed towards their mother was higher when she was in a deteriorated rather than an enhanced nutritional state. This 
result is in line with the assumption of increased offspring begging in broods tended by mothers in poor condition. In this situation, offspring likely increased their begging efforts either cooperatively (e.g. Bell 2007; Madden et al. 2009) or competitively (e.g. Neuenschwander et al. 2003; Smiseth et al. 2003) to elicit sufficient levels of maternal care. Irrespective of the mediating mechanisms, the effects of maternal state on care-related offspring behaviors, as well as on the food transfer among these offspring, illustrate that offspring behaviors during family life are flexible and change according to their mother's condition.

Maternal state did not only shape sibling interactions, but also affected the frequency of care-behaviors mothers expressed towards their offspring. When the nutritional state of mothers was deteriorated (but not when it was enhanced), our data showed that increasing values of maternal body condition at egg hatching entailed a shift from a positive to a negative association between maternal care and brood size. The increase of maternal care with brood size in families tended by females with both low initial body condition and deteriorated nutritional state suggests a trade-off between providing care and somatic maintenance that depends on the number - and thus on the value - of current offspring. Accordingly, females tending small broods might favor somatic maintenance over providing (some forms of) care, e.g. to retain the ability to provide other forms of care such as predator defense (Bateson 1994) or a prospect of future reproduction (Thorogood et al. 2011), whereas females tending large broods might do the opposite (and possibly forfeit chances of future reproduction). In line with this latter reasoning, stitchbird, or hihi (Notiomystis cincta) parents were shown to be largely insensitive to the experimentally enhanced begging displays of their brood when they attempted two 
breeding attempts in one reproductive season, but responded with an increased rate of nestling provisioning when they bred just once (Thorogood et al. 2011).

Surprisingly, the trade-off between providing post-hatching care, somatic maintenance and the prospects of future reproduction seemed to be differently solved by mothers in a deteriorated nutritional state that had featured a high body condition at offspring emergence. Under these conditions, maternal care decreased with brood size. This result could reflect that mothers with a high initial body condition generally favor future over (additional) current reproductive investment (McNamara et al. 2009), but are nevertheless capable of providing high levels of care to small broods without compromising their prospects of future reproduction. Future studies should investigate the nature and adaptive significance of this apparent trade-off between self-maintenance, care and future reproduction. However, our findings reveal that, independent of the underlying mechanisms, females are not forced into this trade-off if their nutritional state is enhanced after offspring emergence.

In summary, we demonstrated that maternal condition did not only influence parental care, but also affected interactions among juveniles and the behaviors they expressed towards their mother. Moreover, we showed that the influence of the mother's current nutritional state on offspring behaviors critically depended on both, her body condition at offspring emergence and the number of offspring she tended. These results thus call for a better integration of female nutritional state and quality in studies on behavioral interactions between parents and offspring as well as among offspring. More generally, our findings illustrate that life-history traits and environmental conditions interact to shape the complex interplay of behaviors characteristic for family life. 


\section{ACKNOWLEDGEMENTS}

We thank the members of "Team Earwig" for their help in the maintenance of animals in the laboratory. This research was supported by the German Science Foundation (DFG; ME4179/1-1 to JM).

\section{REFERENCES}

Alexander RD. 1974. The evolution of social behavior. Annu. Rev. Ecol. Syst. 5:325-383.

Alonso-Alvarez C, Velando A. 2012. Benefits and costs of parental care. In: Royle NJ, Smiseth PT, Kölliker M, editors. The evolution of parental care. Oxford, UK: Oxford University Press. p. 40-61.

Bateson P. 1994. The dynamics of parent-offspring relationships in mammals. Trends Ecol. Evol. 9:399-403.

Bell MB V. 2007. Cooperative begging in banded mongoose pups. Curr. Biol. 17:717-21.

Bleeker M, Kingma SA, Szentirmai I, Székely T, Komdeur J. 2005. Body condition and clutch desertion in penduline tit Remiz pendulinus. Behaviour 142:1465-1478.

Engqvist L. 2005. The mistreatment of covariate interaction terms in linear model analyses of behavioural and evolutionary ecology studies. Anim. Behav. 70:967-971.

Falk J, Wong JWY, Kölliker M, Meunier J. 2014. Sibling cooperation in earwig families provides insights into the early evolution of social life. Am. Nat. 183:547-557.

Fox J. 2003. Effect Displays in R for Generalised Linear Models. J. Stat. Softw. 8:1-18.

Godfray HCJ, Johnstone RA. 2000. Begging and bleating: the evolution of parent-offspring signalling. Philos. Trans. R. Soc. Lond. B. Biol. Sci. 355:1581-91.

Gorman HE, Nager RG. 2003. State-dependent incubation behaviour in the zebra finch. Anim. Behav. 65:745-754.

Hinde CA, Johnstone RA, Kilner RM. 2010. Parent-offspring conflict and coadaptation. Science 327:1373-6.

Kilner RM, Hinde CA. 2012. Parent-offspring conflict. In: Royle NJ, Smiseth PT, Kölliker M, editors. The Evolution of Parental Care. Oxford, UK: Oxford University Press. p. 356. 
Koch LK, Meunier J. 2014. Mother and offspring fitness in an insect with maternal care: phenotypic trade-offs between egg number, egg mass and egg care. BMC Evol. Biol. 14:125.

Kölliker M. 2007. Benefits and costs of earwig (Forficula auricularia) family life. Behav. Ecol. Sociobiol. 61:1489-1497.

Kölliker M, Vancassel M. 2007. Maternal attendance and the maintenance of family groups in common earwigs (Forficula auricularia): a field experiment. Ecol. Entomol. 32:24-27.

Kramer J, Thesing J, Meunier J. 2015. Negative association between parental care and sibling cooperation in earwigs: a new perspective on the early evolution of family life? J. Evol. Biol.

Lamb RJ. 1976 a. Dispersal by nesting earwigs, Forficula auricularia (Dermaptera:

Forficulidae). Can. Entomol. 108:213-216.

Lamb RJ. 1976 b. Parental behavior in the Dermaptera with special reference to Forficula auricularia (Dermaptera: Forficulidae). Can. Entomol. 108:609-619.

Laurien-Kehnen C, Trillmich F. 2004. Maternal food restriction delays weaning in the guinea pig, Cavia porcellus. Anim. Behav. 68:303-312.

Madden JR, Kunc HP, English S, Manser MB, Clutton-Brock TH. 2009. Calling in the gap: competition or cooperation in littermates' begging behaviour? Proc. Biol. Sci. 276:1255-62.

Markman S, Pinshow B, Wright J. 2002. The manipulation of food resources reveals sexspecific trade-offs between parental self-feeding and offspring care. Proc. Biol. Sci. 269:1931-1938.

Mas F, Kölliker M. 2011. Differential effects of offspring condition-dependent signals on maternal care regulation in the European earwig. Behav. Ecol. Sociobiol. 65:341-349.

McNamara JM, Houston Al, Barta Z, Scheuerlein A, Fromhage L. 2009. Deterioration, death and the evolution of reproductive restraint in late life. Proc. R. Soc. B Biol. Sci. 276:40614066.

Meunier J, Kölliker M. 2012. When it is costly to have a caring mother: food limitation erases the benefits of parental care in earwigs. Biol. Lett. 8:547-550.

Meunier J, Wong JWY, Gómez Y, Kuttler S, Röllin L, Stucki D, Kölliker M. 2012. One clutch or two clutches? Fitness correlates of coexisting alternative female life-histories in the European earwig. Evol. Ecol. 26:669-682.

Mock DW, Forbes LS. 1992. Parent-offspring conflict : a case of arrested development. Trends Ecol. Evol. 7:409-413.

Mock DW, Parker GA. 1997. The evolution of sibling rivalry. Oxford, UK: Oxford University Press. 
Morales J, Velando A. 2013 May. Signals in family conflicts. Anim. Behav.

Neuenschwander S, Brinkhof MWG, Kölliker M, Richner H. 2003. Brood size, sibling competition, and the cost of begging in great tits (Parus major). Behav. Ecol. 14:457-462.

Parker G a, Royle NJ, Hartley IR. 2002. Intrafamilial conflict and parental investment: a synthesis. Philos. Trans. R. Soc. Lond. B. Biol. Sci. 357:295-307.

Peig J, Green AJ. 2009. New perspectives for estimating body condition from mass/length data: the scaled mass index as an alternative method. Oikos 118:1883-1891.

Peig J, Green AJ. 2010. The paradigm of body condition: a critical reappraisal of current methods based on mass and length. Funct. Ecol. 24:1323-1332.

Roulin A, Dreiss AN. 2012. Sibling competition and cooperation over parental care. In: Royle NJ, Smiseth PT, Kölliker M, editors. The evolution of parental care. Oxford, UK: Oxford University Press. p. 133-149.

Royle NJ, Hartley IR, Parker GA. 2002. Begging for control: when are offspring solicitation behaviours honest? Trends Ecol. Evol. 17:434-440.

Royle NJ, Smiseth PT, Kölliker M, editors. 2012. The evolution of parental care. Oxford, UK: Oxford University Press.

Schrader M, Jarrett BJM, Kilner RM. 2015. Parental care masks a density-dependent shift from cooperation to competition among burying beetle larvae. Evolution 69:1077-84.

Segers FHID, Gerber B, Taborsky B. 2011. Do maternal food deprivation and offspring predator cues interactively affect maternal effort in fish? Ethology 117:708-721.

Shen S-F, Akçay E, Rubenstein DR. 2014. Group size and social conflict in complex societies. Am. Nat. 183:301-310.

Smiseth PT, Bu RJ, Eikenaes AK, Amundsen T. 2003. Food limitation in asynchronous bluethroat broods: effects on food distribution, nestling begging, and parental provisioning rules. Behav. Ecol. 14:793-801.

Staerkle M, Kölliker M. 2008. Maternal food regurgitation to nymphs in earwigs (Forficula auricularia). Ethology 114:844-850.

Thorogood R, Ewen JG, Kilner RM. 2011. Sense and sensitivity: responsiveness to offspring signals varies with the parents' potential to breed again. Proc. R. Soc. B Biol. Sci. 278:26382645.

Trivers RL. 1972. Parental investment and sexual selection. In: Campbell B, editor. Sexual selection and the descent of man, 1871-1971. Chicago, USA: Heinemann Educational Books. p. 136-179. 
Trivers RL. 1974. Parent-offspring conflict. Am. Zool. 14:249-264.

White J, Leclaire S, Kriloff M, Mulard H, Hatch SA, Danchin E. 2010. Sustained increase in food supplies reduces broodmate aggression in black-legged kittiwakes. Anim. Behav. 79:1095-1100.

Wong JWY, Kölliker M. 2012. The effect of female condition on maternal care in the European earwig. Ethology 118:450-459.

Wong JWY, Kölliker M. 2013. The more the merrier? Condition-dependent brood mixing in earwigs. Anim. Behav. 86:845-850.

Wong JWY, Lucas C, Kölliker M. 2014. Cues of maternal condition influence offspring selfishness. PLoS One 9.

Wong JWY, Meunier J, Lucas C, Kölliker M. 2014. Paternal signature in kin recognition cues of a social insect: concealed in juveniles, revealed in adults. Proc. R. Soc. - Biol. Sci. 281:20141236. 
Manipulation Phase

do

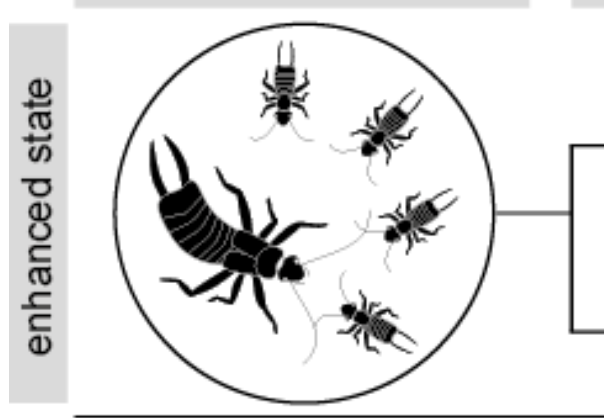

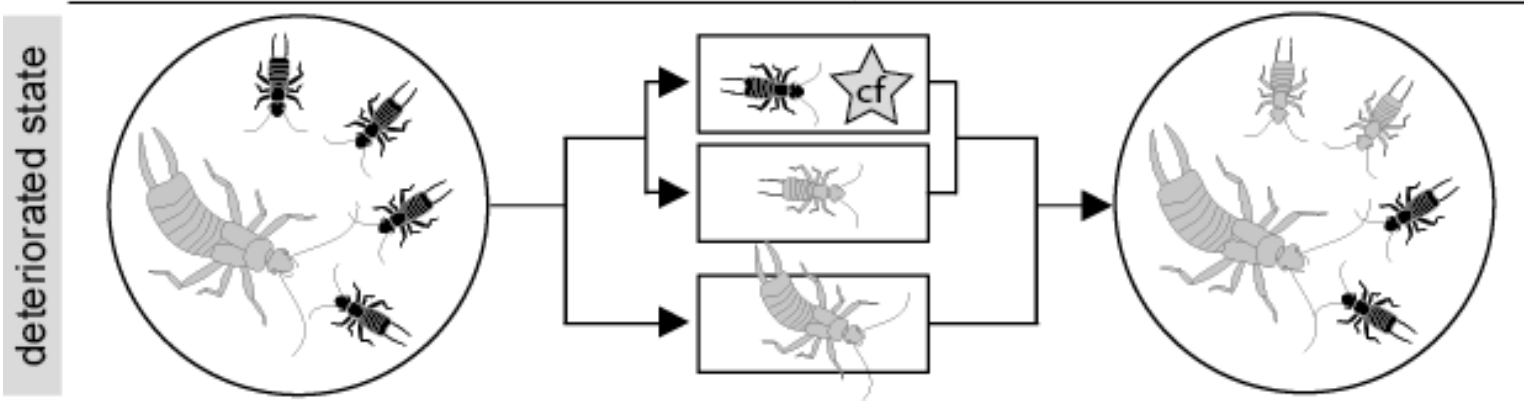

Figure 1: Experimental setup. Female nutritional state was manipulated by selectively feeding (enhanced) or starving (deteriorated) her prior to the behavioral tests. Sibling food transfer (SFT) was measured by providing half of the nymphs (called donor nymphs) with colored food (indicated with "cf"), then reassembling these newly colored nymphs with their remaining, food-deprived siblings (called recipient nymphs) and their mother, allowing family interactions overnight and finally counting the number of recipient nymphs that ingested the colored food provided by the donor nymphs. Grey individuals were fooddeprived in the corresponding experimental phase. 


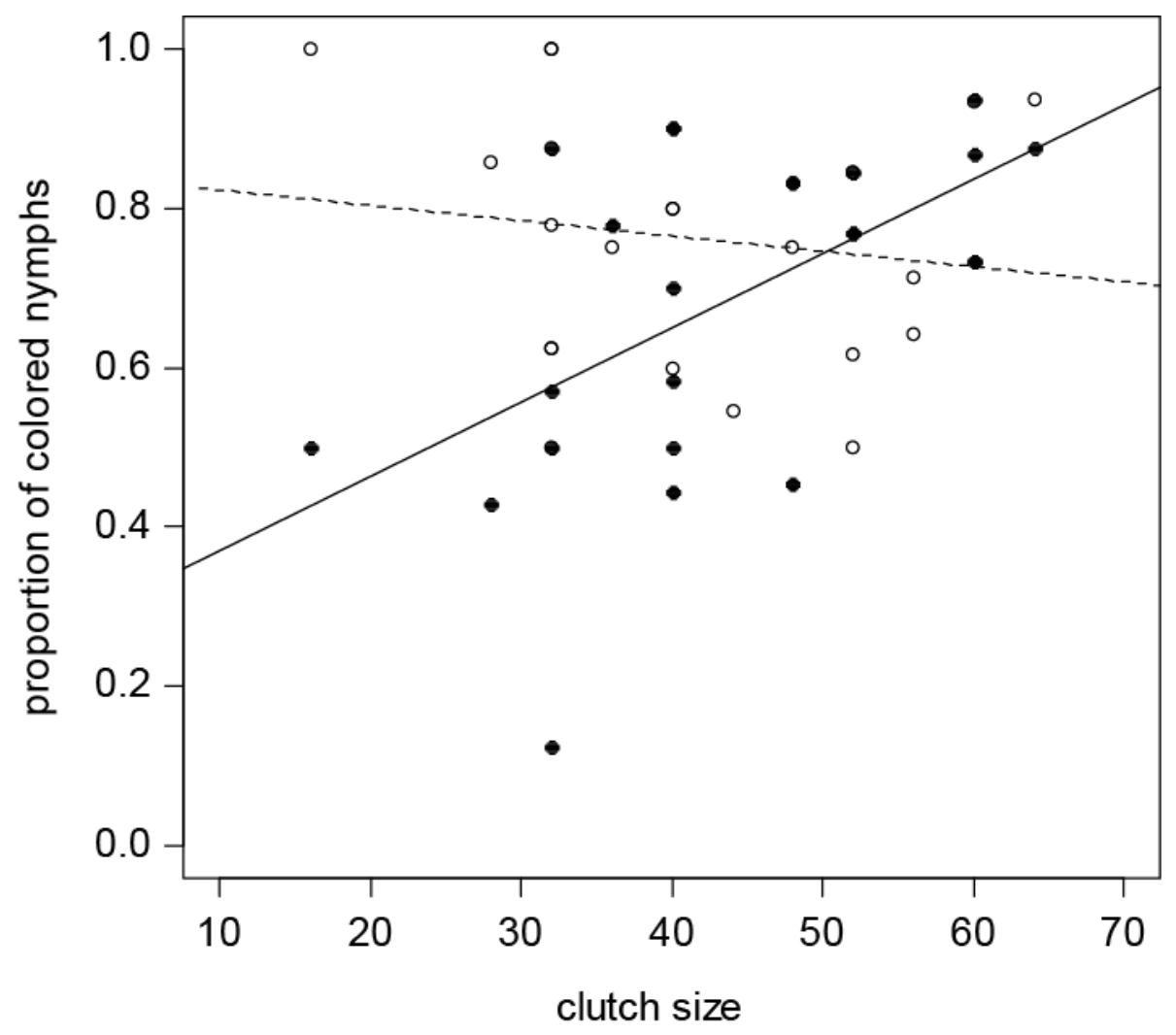

Figure 2: Influence of brood size on the proportion of recipient nymphs that received food during sibling food transfer tests conducted when maternal nutritional state was enhanced (open circles, dashed line) or deteriorated (filled circles, solid line). Note that the discrepancy between the number of visible points and the sample size is due to the overlap of some data points. 


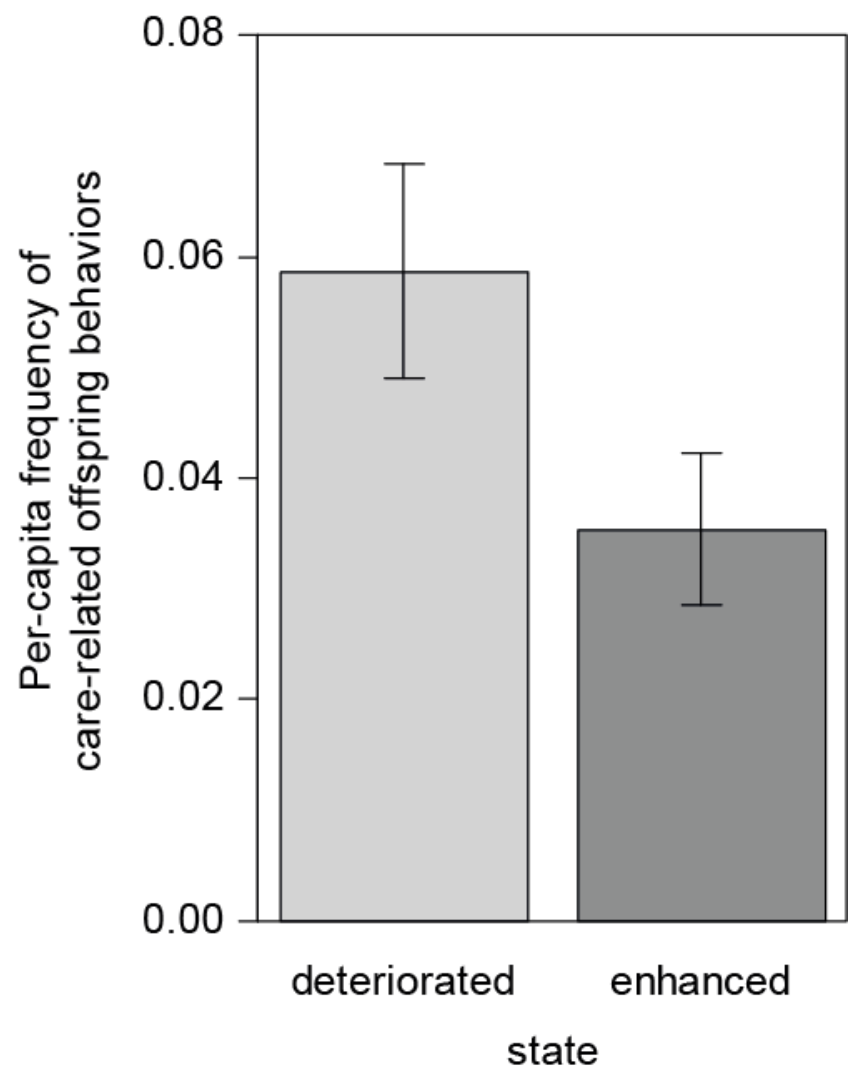

Figure 3: Effects of the mother's nutritional state on the frequency of care-related behaviors expressed by nymphs. 


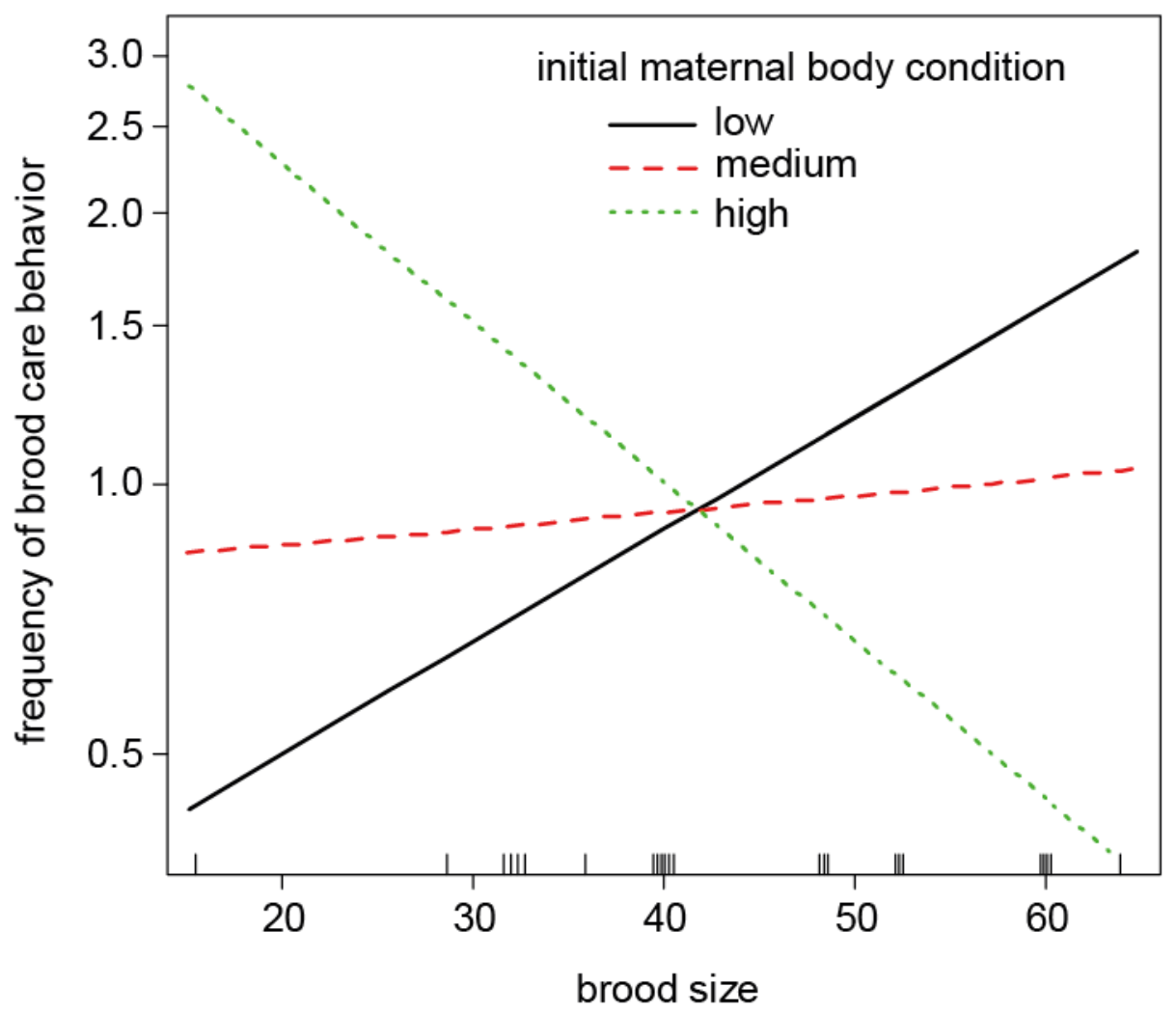

Figure 4: Interacting effect of the initial maternal body condition and brood size on the frequency of care behaviors of mothers in a deteriorated nutritional state. To illustrate the interaction, regression lines are given for an average value of the initial maternal condition (Median $=0.0464$; dashed line) as well as for a comparatively low ( $1^{\text {st }}$ quartile $=0.0442$; solid line) and high ( $3^{\text {rd }}$ quartile $=0.0501$; dotted line) value, respectively . 
Table 1: Effects of the initial body condition of mothers, their brood size and their nutritional state (enhanced or deteriorated) as well as the experimental season on (A) the level of sibling food transfer (measured as the proportion of recipient nymphs that received colored food from their siblings), (B) the frequency of care-related nymph behaviors and (C) the frequency of maternal care behaviors. Note that we report the results of the full models here to facilitate comparisons among them. The (qualitatively unchanged) results of the corresponding reduced models can be found in Table S1. Significant P-values are highlighted in bold print.

\begin{tabular}{lcccccc} 
& \multicolumn{2}{c}{$\begin{array}{c}\text { (A) sibling food } \\
\text { transfer }\end{array}$} & (B) nymph behavior & \multicolumn{2}{c}{ (C) maternal care } \\
\cline { 2 - 7 } & $\chi_{1}{ }^{2}$ & $P$ & $\chi_{1}{ }^{2}$ & $P$ & $\chi_{1}{ }^{2}$ & $P$ \\
\hline initial body condition (IBC) & 0.57 & 0.449 & 0.63 & 0.429 & 0.21 & 0.649 \\
brood size (BS) & 4.49 & $\mathbf{0 . 0 3 4}$ & 0.10 & 0.755 & 1.35 & 0.245 \\
nutritional state (NS) & 0.89 & 0.345 & 3.95 & $\mathbf{0 . 0 4 7}$ & $>0.01$ & 0.937 \\
season & 0.11 & 0.736 & 1.92 & 0.166 & 0.13 & 0.716 \\
IBC:BS & 0.68 & 0.409 & $>0.01$ & 0.933 & $>0.01$ & 0.982 \\
IBC:NS & 0.14 & 0.708 & 0.02 & 0.900 & 1.68 & 0.196 \\
BS:NS & 4.82 & $\mathbf{0 . 0 2 8}$ & 0.14 & 0.707 & 2.99 & 0.084 \\
IBC:BS:NS & 0.20 & 0.653 & 0.24 & 0.624 & 6.24 & $\mathbf{0 . 0 1 3}$ \\
\hline type of model / error family & GLM / binomial & GLM / poisson & GLM / poisson \\
\hline
\end{tabular}

